\title{
Incremental Clustering Algorithm for Earth Science Data Mining
}

\author{
Ranga Raju Vatsavai \\ Computational Sciences and Engineering Division \\ Oak Ridge National Laboratory, Oak Ridge, TN 37831, USA \\ vatsavairr@ornl.gov
}

\begin{abstract}
Remote sensing data plays a key role in understanding the complex geographic phenomena. Clustering is a useful tool in discovering interesting patterns and structures within the multivariate geospatial data. One of the key issues in clustering is the specification of appropriate number of clusters, which is not obvious in many practical situations. In this paper we provide an extension of G-means algorithm which automatically learns the number of clusters present in the data and avoids over estimation of the number of clusters. Experimental evaluation on simulated and remotely sensed image data shows the effectiveness of our algorithm.
\end{abstract}

Keywords: Clustering, EM, GMM, Remote Sensing, K-means, G-means.

\section{Introduction}

Remote sensing, which provides inexpensive, synoptic-scale data with multitemporal coverage, has proven to be very useful in land cover mapping, environmental monitoring, forest and crop inventory, urban studies, natural and man made object recognition, etc. Thematic information extracted from remote sensing imagery is also useful in a variety spatio-temporal applications. For example, land management organizations and the public have a need for more current regional land cover information to manage resources and monitor land use changes. Likewise, intelligence agencies, such as, National Geospatial Intelligence Agency (NGA), and Department of Homeland Security (DHS), utilizes pattern recognition and data mining techniques to classify both natural and man made objects from large volumes of high resolution imagery.

Clustering algorithms play a key role in earth science data mining. They are often used to analyze complex and large volumes of multivariate geospatial data, such as, remotely sensed images, sensor measurements, field observations, etc., as a first step in gaining insights into the structure or natural groupings. Clustering is also used to in compression, exploratory analysis, and summarization of the data. Cluster analysis is used in many other spatial and spatiotemporal application domains. Cluster analysis is routinely used in epidemiology for finding unusual groups of health-related events. Cluster analysis is also used in detection of crime hot spots.

G. Allen et al. (Eds.): ICCS 2009, Part II, LNCS 5545, pp. $375-3842009$.

(C) Springer-Verlag Berlin Heidelberg 2009 
One of the key challenges in clustering is the specification of the number of clusters. Determining an optimal number of clusters manually is not feasible given the complexity and volume of geospatial data sets. In this paper we provide a simple extension of the G-means 3 algorithm that automatically discovers the number of clusters. Experimental evaluation shows that our algorithm can avoid the common problem of ending up with a large number of spurious clusters by the G-means algorithm.

\section{Related Work and Our Contributions}

Clustering is a fertile research area with applications cutting across many domains. A large number of clustering algorithms can be found in the literature [45. These algorithms can be broadly categorized into: hierarchical, partitional, density-based, and grid-based methods. Partitional clustering algorithms, especially, K-Means algorithm is very popular in several application domains, including earth sciences. One of the key inputs to K-Means algorithm is the specification of $\mathrm{K}$, the number of clusters. However, determining an optimal number of clusters manually is not feasible given the complexity and volume of geospatial data sets.

Considerable research has gone into finding the optimum number of clusters directly from the data itself 267/813. In [13] authors proposed a G-means algorithm that automatically discovers the number of clusters. Basic idea behind G-means is simple. Initial number of clusters (k) determined by k-means are incremented by splitting each cluster that doesn't pass a statistical test. The clustering process is repeated until all the clusters have passed this statistical test. In many practical situations there is a danger of over estimating the number of clusters, especially if the model is assumed to be a Gaussian Mixture Model (GMM). We extended the G-means algorithm to overcome this practical limitation. In many situations G-means clustering algorithm tends to find more clusters. In order to reduce the chance of finding more clusters, we devised a new approach that prevents some splits and allows to reverse the splits. In a nutshell we made two modifications to the G-means algorithm. First, instead of univariate test statistic, we used a multivariate test statistic, known as, ShapiroWilk statistic. This modification has following advantages. First we don't have to project multivariate data into 1-d. This is important for earth science data mining as the geospatial data is often high-dimensional in nature. Finding a good projection can be as difficult as finding a good K. Second, AD test is good for small samples, that is, number of samples $\leq 25$. However, in earth science data sets typically we have large number of samples (per cluster). Finally, the multivariate Shapiro-Wilk test exhibits good power against alternatives [10. We used KL Divergence measure after splitting the clusters to see if any pair of clusters are too close to each other. If any two clusters are too close to each other, then it is better to combine them, even though such combination may violate significance testing. In the following sections, we present our algorithm and experimental results. 


\section{Clustering Framework}

Basic statistical framework for our clustering approach is Gaussian Mixture Models (GMMs). Typically model based clustering approaches are not applied on entire data set given the computational and data complexity. Rather a subset of data samples are collected from the full data set. Model parameters are estimated using these samples. Once a model is constructed, all the samples (data points) in the full data set can then be assigned to one of the clusters, based on some distance (or decision) criteria. Our algorithm is based on the assumption that the data samples are generated by a GMM. Then the objective is to learn the GMM parameters from these samples. We now briefly describe an expectation maximization based algorithm to learn the GMM parameters.

\subsection{Estimating GMM Parameters}

Let us now assume that the sample data set $D=\left\{x_{i}\right\}_{i=1}^{n}$ is generated by the following mixture density.

$$
p\left(x_{i} \mid \Theta\right)=\sum_{j=1}^{K} \alpha_{j} p_{j}\left(x_{i} \mid \theta_{j}\right)
$$

Here $p_{j}\left(x_{i} \mid \theta_{j}\right)$ is the pdf corresponding to the mixture $j$ and parameterized by $\theta_{j}$, and $\Theta=\left(\alpha_{1}, \ldots, \theta_{K}, \theta_{1}, \ldots, \theta_{K}\right)$ denotes all unknown parameters associated with the $K$-component mixture density. For a multivariate normal distribution (eq. 2), $\theta_{j}$ consists of elements of the mean vectors $\mu_{j}$ and the distinct components of the covariance matrix $\Sigma_{j}$.

$$
p\left(x \mid y_{j}\right)=\frac{1}{\sqrt{(2 \pi)^{-N}\left|\Sigma_{j}\right|}} e^{\frac{-1}{2}\left(x-\mu_{j}\right)^{t}\left|\Sigma_{j}\right|^{-1}\left(x-\mu_{j}\right)}
$$

The log-likelihood function for this mixture density can be defined as:

$$
L(\Theta)=\sum_{i=1}^{n} \ln \left[\sum_{j=1}^{M} \alpha_{j} p_{j}\left(x_{i} \mid \theta_{j}\right)\right] .
$$

In general, Equation 3 is difficult to optimize because it contains the ln of a sum term. However, this equation greatly simplifies in the presence of unobserved (or incomplete) samples. Typically, we assume that the cluster labels as missing (unobserved) data, and use expectation maximization technique to estimate parameters $(\Theta)$. The EM algorithm consists of two steps, called the E-step and and M-step as given below.

E-Step. For multivariate normal distribution, the expectation $E[$.$] , which is$ denoted by $p_{i j}$, is the probability that Gaussian mixture $j$ generated the data point $i$, and is given by:

$$
p_{i j}=\frac{\left|\hat{\Sigma}_{j}\right|^{-1 / 2} e^{\left\{-\frac{1}{2}\left(x_{i}-\hat{\mu}_{j}\right)^{t} \hat{\Sigma}_{j}^{-1}\left(x_{i}-\hat{\mu}_{j}\right)\right\}}}{\sum_{l=1}^{M}\left|\hat{\Sigma}_{l}\right|^{-1 / 2} e^{\left\{-\frac{1}{2}\left(x_{i}-\hat{\mu}_{l}\right)^{t} \hat{\Sigma}_{l}^{-1}\left(x_{i}-\hat{\mu}_{l}\right)\right\}}}
$$


Table 1. Algorithm for Computing Parameter of Finite Gaussian Mixture Model Over Unlabeled Training Data

Inputs: $D$, sample data set; $\mathrm{K}$, the number of clusters.

Initial Estimates: Do clustering by K-Means, and estimate initial parameter using Maximum Likelihood Estimation (MLE) technique to find $\hat{\theta}$.

Loop: While the complete data log-likelihood improves:

E-step: Use current classifier to estimate the class membership of each unlabeled sample, i.e., the probability that each Gaussian mixture component generated the given sample point, $p_{i j}$ (see Equation 4).

M-step: Re-estimate the parameter, $\hat{\theta}$, given the estimated Gaussian mixture component membership of each unlabeled sample (see Equations 5. 6. (7)

Output: Parameter vector $\Theta$.

M-Step. The new estimates (at the $k^{\text {th }}$ iteration) of the model parameters in terms of the old parameters are computed using the following update equations:

$$
\begin{gathered}
\hat{\alpha}_{j}^{k}=\frac{1}{n} \sum_{i=1}^{n} p_{i j} \\
\hat{\mu}_{j}^{k}=\frac{\sum_{i=1}^{n} x_{i} p_{i j}}{\sum_{i=1}^{n} p_{i j}} \\
\hat{\Sigma}_{j}^{k}=\frac{\sum_{i=1}^{n} p_{i j}\left(x_{i}-\hat{\mu}_{j}^{k}\right)\left(x_{i}-\hat{\mu}_{j}^{k}\right)^{t}}{\sum_{i=1}^{n} p_{i j}}
\end{gathered}
$$

The EM algorithm iterates over these two steps until convergence is reached. We can now put together these individual pieces into the following algorithm (Table 1) which computes the parameters for each component in the finite Gaussian mixture model that generated our sample data $D$ (without any cluster labels).

\subsection{Simulation Example 1}

We now demonstrate GMM clustering algorithm (1) on a simulated data set. We generated a GMM with three components. The parameters are given in Table 2 and Table 3 . We generated 150 bivariate Gaussian samples from each

Table 2. Simulation Parameters (Mean)

Table 3. Simulation Parameters

\begin{tabular}{|c|c|c|}
\hline C1 & $\mathrm{C} 2$ & C3 \\
\hline $\mathrm{x}$ & $\mathrm{x}$ & $\mathrm{x}$ \\
\hline $\mathrm{x} 30.0025 .00$ & 60.0040 .00 & 60.0050 .00 \\
\hline y 25.0040 .00 & 40.0090 .00 & 50.0070 .00 \\
\hline
\end{tabular}
(Covariance)

\begin{tabular}{rr}
\hline \multicolumn{1}{c}{$y$} \\
\hline C1 55.0025 .00 \\
C2 80.0050 .00 \\
C3 50.0040 .00 \\
\hline
\end{tabular}


component density. We applied GMM clustering algorithm on this sample data set by assuming different $K$ values and the results were summarized in Figure 1 From the figure it can be seen that when $K$ assumption is correct (that is, $K=3$ ) we have very good estimates (compare subfigures (a) and (c)), however for other K's (subfigures (b) and (d)) the estimates are very different than the original distribution. This simulation emphasizes the need to estimate a good $\mathrm{K}$ value (if possible automatically from the data).

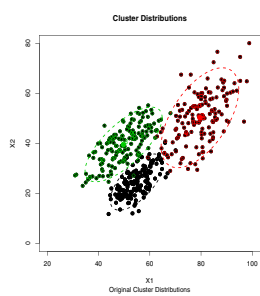

(a) Simulated $(\mathrm{K}=3)$

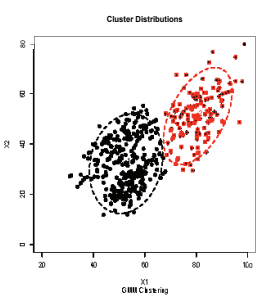

(b) Estimated $(\mathrm{K}=2)$

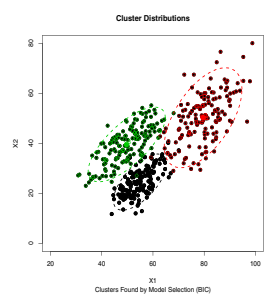

(c) Estimated $(\mathrm{K}=3)$

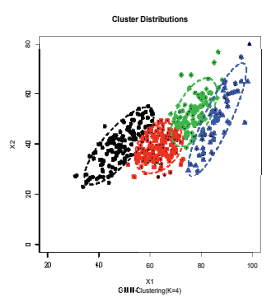

(d) Estimated $(\mathrm{K}=4)$

Fig. 1. Simulated vs. Estimated (GMM-Clustering for different K values)

\section{Learning to Estimate K}

In this section we address the problem of estimating $\mathrm{K}$ automatically from the data. As with the estimation of the model parameters for finite Gaussian mixture model, we assume that the training dataset $D$ is generated by a finite Gaussian mixture model, but we don't know either the number of components or the labels for any of the mixture component. In the previous section, we devised an algorithm to find parameters by assuming a $K$-component finite Gaussian mixture model. In general, we can estimate parameters for any arbitrary $K$-component model, as long as there are sufficient number of samples available for each component and the covariance matrix does not become singular. Then the question remains, which $K$-component model is better? This question is addressed in the area of model selection, where the objective is to chose a model that maximizes a cost function. There are several cost functions available in the literature, most commonly used measures are Akaike's information criterion (AIC), Bayesian information criteria (BIC), and minimum description length (MDL). The common criteria behind these models is to penalize the models with additional parameters, so BIC and AIC based model selection criteria follows the principal of parsimony. In this study we considered BIC as a model selection criteria, which also takes the same form as MDL. We also chose BIC, as it found to be very useful in model based clustering [2], and also because it is defined in terms of maximized log-likelihood which any way we are computing in our parameter estimation procedure defined in the previous section. BIC can be defined as

$$
B I C=M D L=-2 \log L(\Theta)+m \log (N)
$$

where $N$ is the number of samples and $m$ is the number of parameters. We now describe our BIC based model selection criteria to determine the number 
components in each aggregate class. First, we take the aggregate class and split it into two Gaussians at a time using the Gaussian splitting criteria specified in [11. Then the parameters of this new mixture model are estimated using the algorithm 1. This process is recursively applied for a fixed number times or BIC is minimized.

On the other hand, G-means [3] clustering is initialized by k-means clustering for suitable initial $\mathrm{K}$ value. Each cluster is then tested for normality using univariate test: Anderson-Darling (AD) statistic. For a user given p-value, if AD test fails, then the cluster is split into two clusters. K-means cluster is performed again with new $\mathrm{K}$, and the process is repeated until no more (new splits) clusters can be found. Multivariate data is projected on to 1-d to facilitate AD test. In 3], authors argued that BIC has a tendency to find more clusters. In our experiments, we found that G-means clustering also tend to find more clusters. We demonstrate this through an example simulated data set. In order to reduce the chance of finding more clusters, we devised a new approach that prevents splits and allows to reverse the splits.

Our algorithm differs from G-means in two ways. First, instead of univariate test statistic, we used a multivariate test statistic, known as, Shapiro-Wilk statistic. More details on Shapiro-Wilk test can be found in [12. This modification has following advantages. First we don't have to project multivariate data into 1-d. This is important for earth science data mining as the geospatial data is often high-dimensional in nature. Finding a good projection can be as difficult as finding a good K. Second, AD test is good for small samples, that is, number of samples $\leq 25$. Finally, the multivariate Shapiro-Wilk test exhibits good power against alternatives [10. Finally, statistical tests are sensitive to noise. It is likely that splitting process (increasing $\mathrm{K}$ ) continue beyond optimal $\mathrm{K}$ as many times statistical significance test fails (even though clusters are close to multivariate normal). As a check to prevent this happening, we added additional criteria to check for the quality of splits. We used KL Divergence [9] measure after splitting to see if any pair of clusters are too close to each other. If any two clusters are too close to each other, then it is better to combine them. The new algorithm (GMM-Adaptive-K) is summarized in Table 4.

\subsection{Simulation Example 2}

We now demonstrate GMM-Adaptive-K clustering algorithm (44) on the simulated data set (Table 3). The results were summarized in Figure2, First iteration found two clusters (Figure 2(b)), red cluster passes Shapiro-Wilk test. As result, only the 2nd cluster (black) is split into two clusters (c). In the next iteration, red cluster failed Shapiro-Wilk test, as a result it was split into two clusters (d). The G-means cluster algorithm would have resulted in a final solution shown in Figure 2(e). On the other-hand the additional step introduced in our algorithm, finds that these two clusters are very close (KL-Divergence), thus decrements number of clusters to 3. Final solutions is shown in Figure 2(f). Compare Figure 2(f) with original distribution in Figure 2(a). 


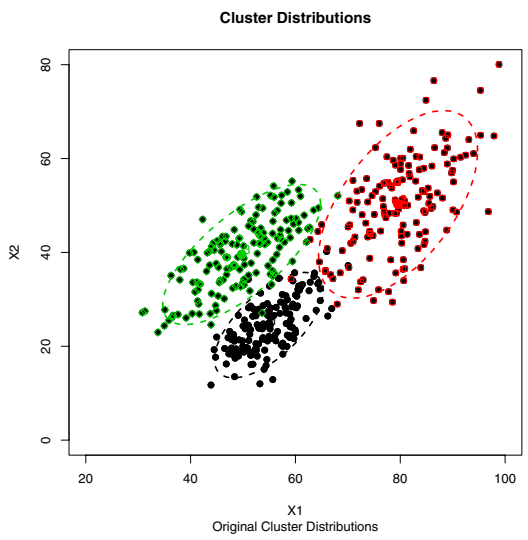

(a) Original Distribution

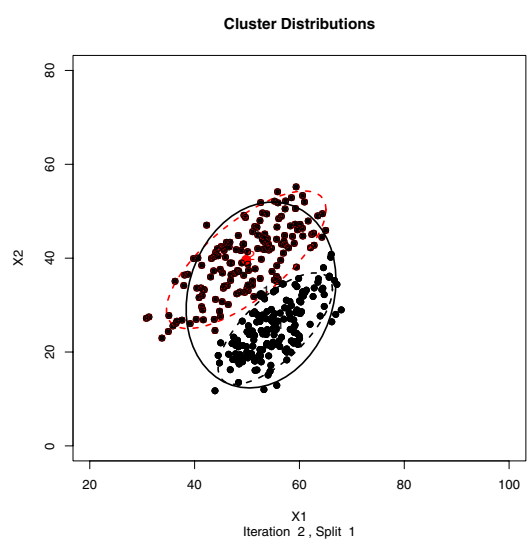

(c) Iteration 2, Split 1

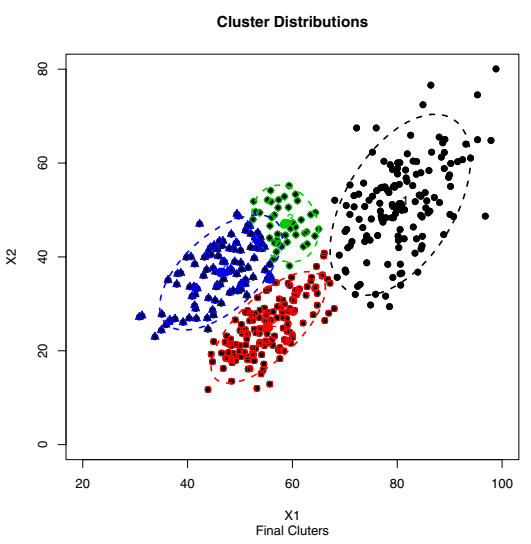

(e) Without Merge Step

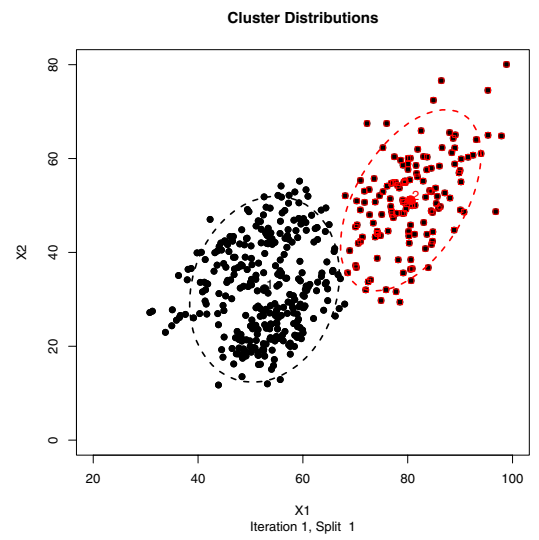

(b) Iteration 1 , Split 1

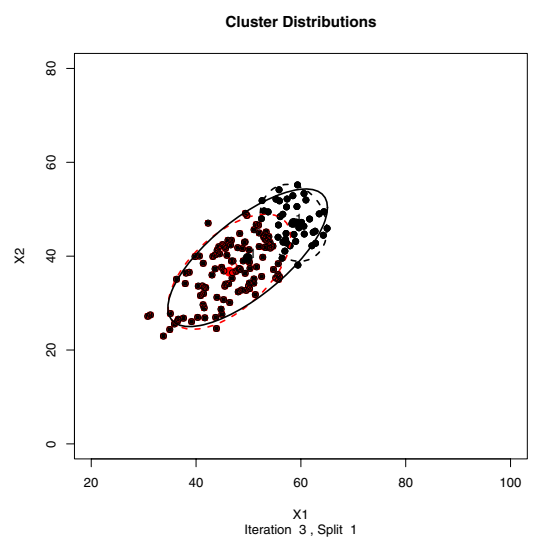

(d) Iteration 3, Split 1

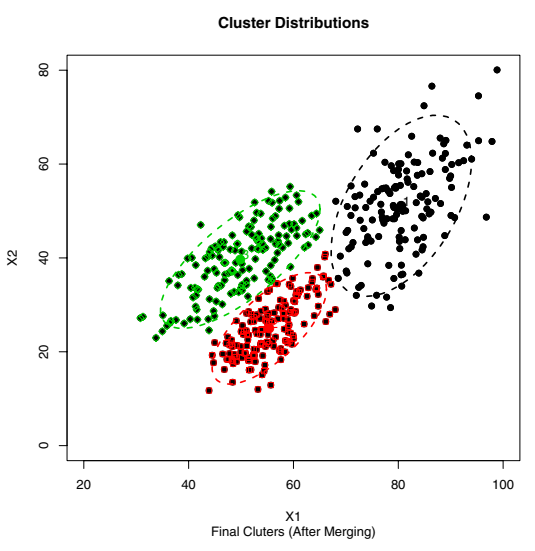

(f) With Merge Step

Fig. 2. GMM-Apdaptive-K Algorithm Trace 
Table 4. GMM-Adaptive-K Algorithm

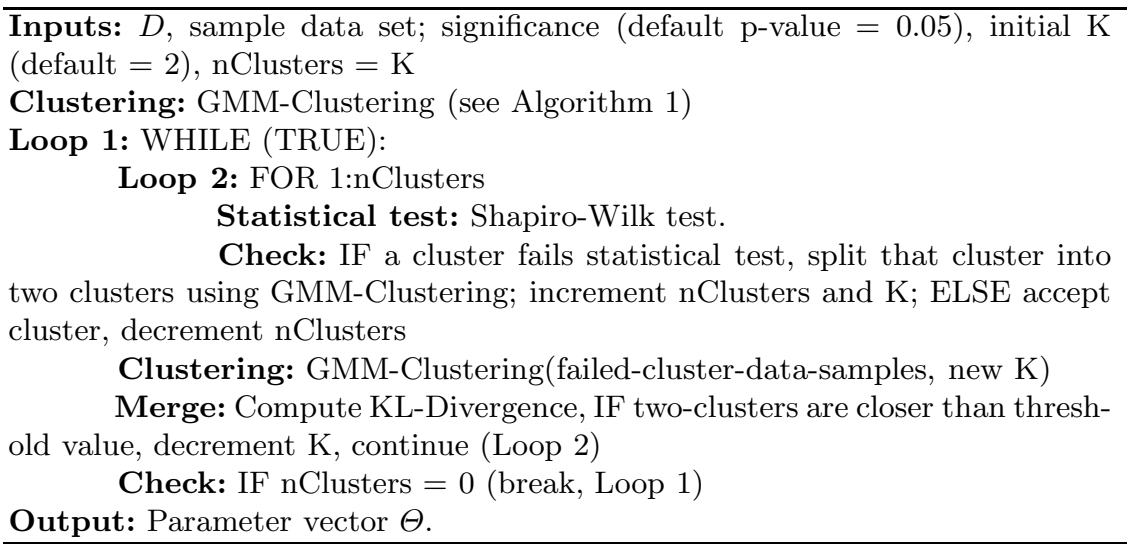

\section{Experimental Results}

We have applied our GMM-Adaptive-K algorithm on the real data set described below.The Cloquet study site encompasses Carlton County, Minnesota, which is approximately 20 miles southwest of Duluth, Minnesota. The region is predominantly forested, composed mostly of upland hardwoods and lowland conifers. There is a scattering of agriculture throughout. The topography is relatively flat, with the exception of the eastern portion of the county containing the St. Louis River. Wetlands, both forested and non-forested, are common throughout the area. The largest city in the area is Cloquet, a town of about 10,000. We used a spring Landsat 7 scene, taken May 31, 2000, and clipped to the study region. The final rectified and clipped image size is 1343 lines x 2019 columns x

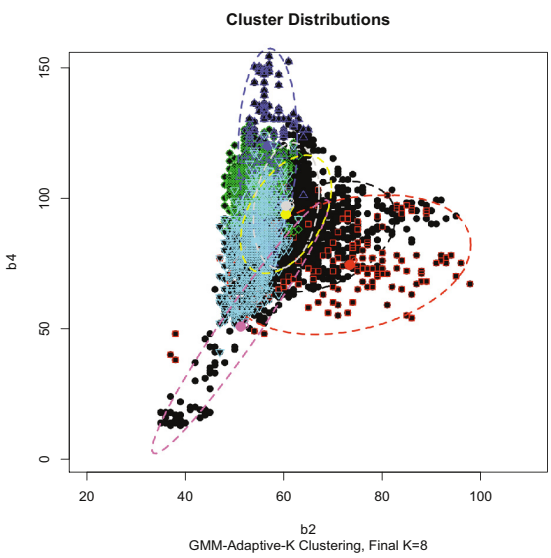

(a) Prob. Distributions of Clusters

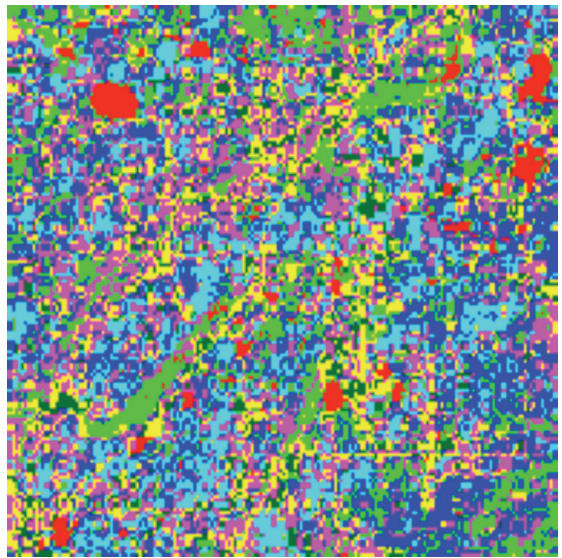

(b) Clustered Image Subset

Fig. 3. GMM-Apdaptive-K Algorithm on Carleton Satellite Image Data 
6 bands. We selected 400 random plots. From each plot, we extracted 9 feature vectors ( 6 dimensional) by placing a $3 \times 3$ window at the center of each plot. That is, sample data set consisted of of 3600 feature vectors. We applied our GMM-Adaptive-K algorithm on this sample data set and found 8 clusters. In a supervised classification experiment, remote sensing analysts have identified 10 classes for this study site. Supervised classification image was visually compared with our clustering algorithm. It appears a good correspondence between the clusters and thematic classes identified by the analyst. However, further analysis and experimentation is needed to establish this correspondence between the clusters and thematic (information) clusters. Figure 3(a) shows the cluster (bivariate density) distributions in feature space (bands 2 and 4), and Figure 3 (b) shows a small clip from the clustered image.

\section{Conclusions}

We developed an incremental clustering algorithm. The algorithm is based on GMM distribution and expectation maximization (EM) parameter estimation. The algorithm is also an extension of G-means algorithm, which splits clusters failing statistical significance tests, in a iterative manner to find optimal number of clusters. However, our algorithm avoids an important limitation of over estimation of $\mathrm{K}$ by employing KL divergence measure to find highly overlapping clusters and try to avoid them from further splitting. Experimental evaluation on simulated data shows that our algorithm produces parameters which are very close to the original distribution. Clustering on a real data set shows a good correspondence between the clusters and thematic (information) classes chosen by the remote sensing analyst in a supervised classification project. Further analysis and experimentation is needed to understand the performance and utility of this algorithm in earth science data mining applications.

\section{Acknowledgments}

We would like to thank our former collaborators Thomas E. Burk, Jamie Smedsmo, Ryan Kirk and Tim Mack at the University of Minnesota for useful comments and inputs into this research. The comments of Eddie Bright, Phil Coleman, and Veeraraghavan Vijayraj, have greatly improved the technical accuracy and readability of this paper. Prepared by Oak Ridge National Laboratory, P.O. Box 2008, Oak Ridge, Tennessee 37831-6285, managed by UT-Battelle, LLC for the U. S. Department of Energy under contract no. DEAC05-00OR22725.

\section{References}

1. Feng, Y., Hamerly, G.: Pg-means: learning the number of clusters in data. In: Advances in Neural Information Processing Systems 19, pp. 393-400. MIT Press, Cambridge (2007)

2. Fraley, C., Raftery, A., Wehrens, R.: Incremental model-based clustering for large datasets with small clusters. Journal of Computational and Graphical Statistics 14 (2005) 
3. Hamerly, G., Elkan, C.: Learning the k in k-means. In: Neural Information Processing Systems. MIT Press, Cambridge (2003)

4. Jain, A.K., Dubes, R.C.: Algorithms for clustering data. Prentice-Hall, Inc., Upper Saddle River (1988)

5. Jain, A.K., Murty, M.N., Flynn, P.J.: Data clustering: a review. ACM Comput. Surv. 31(3), 264-323 (1999)

6. Mclachlan, G.J., Peel, D.: On a resampling approach to choosing the number of components in normal mixture models. In: Proceedings of Interface 96, 28th Symposium on the Interface, pp. 260-266 (1997)

7. Carreira-Perpi, M.A.: Mode-finding for mixtures of gaussian distributions. IEEE Trans. Pattern Anal. Mach. Intell. 22(11), 1318-1323 (2000)

8. Pelleg, D., Moore, A.W.: X-means: Extending k-means with efficient estimation of the number of clusters. In: ICML 2000: Proceedings of the Seventeenth International Conference on Machine Learning, pp. 727-734. Morgan Kaufmann Publishers Inc, San Francisco (2000)

9. Penny, W.: Kullback-liebler divergences of normal, gamma, dirichlet and wishart densities (2001)

10. Rivas, M.: An exposition on tests for multivariate normality (2007)

11. Sankar, A.: Experiments with a gaussian merging-splitting algorithm for hmm training for speech recognition. In: Proceedings of the Broadcast News Transcription and Understanding Workshop, pp. 99-104 (1998)

12. Shapiro, S.S., Wilk, M.B.: An analysis of variance test for normality (complete samples). Biometrika 3(52) (1965)

13. Tibshirani, R., Walther, G., Hastie, T.: Estimating the number of clusters in a data set via the gap statistic. Journal of the Royal Statistical Society: Series B (Statistical Methodology) 63(2), 411-423 (2001) 